\title{
Joint Motion Control of a Powered Lower Limb Orthosis for Rehabilitation
}

\author{
Nelson Costa, Milan Bezdicek, Michael Brown, John O. Gray, Darwin G. Caldwell* \\ Centre for Robotics and Automation, University of Salford, Manchester M5 4WT, UK \\ Stephen Hutchins \\ Directorate for Prosthetics and Orthotics, University of Salford, Manchester M5 4WT, UK
}

\begin{abstract}
Many patients with spinal injures are confined to wheelchairs, leading to a sedentary lifestyle with secondary pathologies and increased dependence on a carer. Increasing evidence has shown that locomotor training reduces the incidence of these secondary pathologies, but the physical effort involved in this training is such that there is poor compliance. This paper reports on the design and control of a new "human friendly" orthosis (exoskeleton), powered by high power pneumatic Muscle Actuators (pMAs). The combination of a highly compliant actuation system, with an intelligent embedded control mechanism which senses hip, knee, and ankle positions, velocity, acceleration and force, produces powerful yet inherently safe operation for paraplegic patients. This paper analyzes the motion of ankle, knee, and hip joints under zero loading, and loads which simulate human limb mass, showing that the use of "soft" actuators can provide a smooth user friendly motion. The application of this technology will greatly improve the rehabilitative protocols for paraplegic patients.
\end{abstract}

Keywords: Exoskeleton, human-centred robotics, Human Adaptive Mechatronics, pneumatic Muscle Actuators (pMAs), rehabilitation.

\section{Introduction}

Although reciprocal walking for paraplegic patients with complete thoracic lesions has been routinely available since the early 1980 s, many patients with spinal injuries still remain permanently confined to a wheelchair ${ }^{[1]}$. However, research has shown that the ability to stand and walk;

i) decreases the instance and severity of secondary problems, including the formation of contractures in lower limbs, pressure sores, bowl infections, lower limb spasticity, osteoporosis, and kidney and urinary tract infections;

ii) reduces patient dependence on a carer;

iii) improves cardiopulmonary function; and

iv) has a positive psychological effect which impacts on the rehabilitation process, attempts to gain employment, and family and social life ${ }^{[1]}$.

Locomotor training following neurological injury has been shown by many studies to have therapeutic benefits. However, treatment relies on physiotherapy procedures that are extremely labour intensive. In addition, these training and rehabilitation activities place high levels of physical strain on a patient, and the effort

\footnotetext{
Manuscript received December 14, 2005; revised January 16, 2006.

${ }^{*}$ Corresponding author. E-mail address:

D.G.Caldwell@salford.ac.uk
}

required often leads to low levels of compliance. All this must be achieved in an environment in which there is a global shortage of staff with appropriate training ${ }^{[1 \sim 3]}$.

One alternative to manual locomotor training is the use of powered limb orthoses (exoskeleton), to provide mechanical assistance during standing and walking. Two approaches to powered locomotor training have evolved.

\section{i) Functional electrical stimulation}

Functional electrical stimulation (F.E.S), involves external electrical stimulation of leg muscles with the aim of generating standing and walking motions. During exercise, support is usually provided by an orthosis ${ }^{[4]}$. Steady progress is being made using this technique, although there are still side effects such as skin irritation (which can be addressed), and fatigue (which is more difficult to address). It is estimated that this technique may require more than 9 times the energy consumption of "normal" walking, due to the over stimulation of anatomical muscle groups, and subsequent unsuitably high torque production ${ }^{[5]}$. The effort required from patients, means that compliance is low ${ }^{[6]}$.

\section{ii) Externally powered orthosis}

Externally powered orthoses are typically driven by electric motors with several recent devices having been developed primarily for hospital based rehabilitation. Both the Lokomat, which is a 4 dof (degrees of freedom) 
per leg treadmill based system, and the Fraunhofer walker, which provides 3 dof per leg in a crank slider motion, with rotation for the ankle ${ }^{[7]}$, have achieved some success using this approach.

With these externally powered systems, safety is of course a critical issue, with each system having a number of safety interlocks. However, it has been suggested that where possible, extending the safety concept from monitoring and control to form part of the design philosophy could be valuable; particularly where humans and mechanical systems operate in close proximity. It has been suggested that this "softer" design philosophy should seek to address actuation and structural material issues.

In recent years, there has been increasing interest in the emerging field of human-centred robotics, or $\mathrm{Hu}-$ man Adaptive Mechatronics ${ }^{[8 \sim 17]}$. This area involves studies of close interactions between robotic systems and humans, including direct human-robot contact. In such applications, traditional figures of merit such as bandwidth, peak force or torque, and work envelope, do not fully define requirements; and specifically do not address safety requirements.

For any robot or mechanical system, safety is dependent on a mechanism's mechanical, electrical, and software design characteristics. However, the biggest danger when working in close proximity to robots/mechanisms is the potential for impact resulting from the large effective inertia (effective impedance). It is therefore suggested that if inherent safety is to be achieved, it is necessary to design mechanisms that have naturally low impedance, and then couple this with robust electrical and software safety systems.

This paper reports on the design of a new "human friendly" orthosis, powered by high power pneumatic Muscle Actuators (pMAs) that benefit from inherent softness in their construction. Initial sections provide a background introduction to the actuators, their operation and benefits. Section 3 shows our mechanical design, followed by a consideration of system control and operation. Experimental results then provide extensive testing of the operation and control of joints under unloaded and simulated load conditions. The paper finishes by drawing conclusions based on this work, and suggests paths for the future.

\section{Actuator design}

Actuators and actuation systems are essential parts of all mechatronic structures, providing the forces, torques, and mechanical motions needed to move joints, limbs, or a body. To provide safety in human centred robotics, work at the University of Salford has focused on the use of pMAs (based on an adaptation of a braided pneumatic actuation design dating back to at least the 1920s) which has led to the development of a range of actuators with enhanced performance characteristics ${ }^{[18]}$.

The actuator used in these designs, consists of an inner liner with a tubular braided mesh outer. When air within the actuator is pressurized, it contracts by $30 \sim 35 \%$, and depending on the muscle diameter can produce a contractile force of up to $7000 \mathrm{~N}$ from a muscle with a $70 \mathrm{~mm}$ nominal diameter, and a weight of less than 100g. Detailed construction, operation, and mathematical analysis of these actuators can be found in $[19,20]$. The key features that make this actuation technique suitable for powered assistive devices, are:

- Muscles can be produced in a range of lengths and diameters, and are simple to manufacture.

- Muscles have an extremely high power to weight ratio.

- Muscles contract by $30 \sim 35 \%$ of their dilated length, comparable with natural muscle.

- 'Soft' construction and finite maximum contraction make pMAs safe for human-machine interaction.

- Muscles can be controlled to a displacement accuracy of $1 \%$, and can have a bandwidth of $5 \mathrm{~Hz}$ when operating as part of an antagonist pair.

- Compared with natural muscle, pMAs provide up to 10 times more force for an equivalent cross-sectional area.

Having noted that pMAs have inherent capacity to modulate compliance/impedance, and the potential to produce a biologically inspired actuator combining many if not all of the best features of natural and technological science so far, the next developmental stage was to incorporate these actuators into a lower body exoskeleton prototype orthosis.

\section{Mechanical design concepts}

Our mechanical structure used to form an orthosis to assist those with paralysis or muscle wastage, consists of a 10 Degree of Freedom mechanism (5DoF for each leg), although only 8 DoF are currently powered; corresponding to the fundamental natural motion and range of human legs from the hip to the ankle, but excluding less significant shin/calf movements. The hip structure has 3 DoF in total (flexion/extension, abduction-adduction, and lateral-medial rotation, however, the last degree of freedom is not co-axial, and is currently unpowered). 1 DoF at the knee permits flexion/extension of the lower leg, and $1 \mathrm{DoF}$ at the ankle for dorsiflexion/plantar flexion. These joint coordinate conventions are represented in Fig. 1, together with the average mass and centre of gravity for a standard human. 

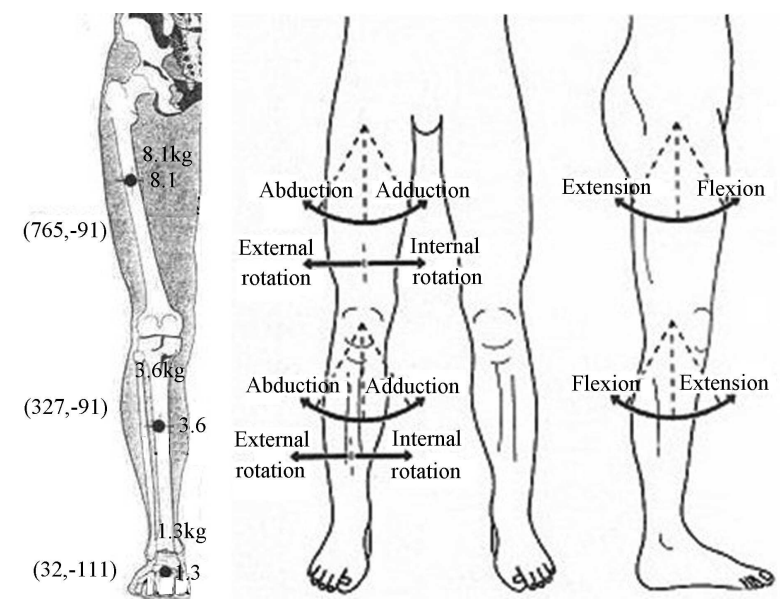

Fig. 1 Average mass in $\mathrm{kg}$, centre of gravity of limbs, and joint coordinate conventions

The leg structure is constructed primarily from aluminium, with joint sections fabricated in steel using precision mechanics. Support for the thighs and calves are provided by moulded structures tailored to the anatomy of the patient. These moulded structures are mounted on the aluminium structures, as shown in Fig. 2. The total weight of the exoskeleton, consisting of both legs and a rigid spine (on to which the muscles for hip flexion/extension and abduction/adduction are mounted), is $11 \mathrm{~kg}$. The length from hip to knee is $520 \mathrm{~mm}$, with $500 \mathrm{~mm}$ from the ankle's base to the top of the knee.

As with all electrical systems, it must be recognised that this mass does not include a power source, but does include all valves and electrical control systems. The power source is compressed air, which is readily and safely available in most hospitals and clinics.

The compact actuator structure allows for integration as close as possible to the respective powered joints. Ankle actuators (two actuators), are mounted at the side of the calf, while actuators for the knee and leg hip rotation actuators (two actuators), are mounted on the lateral surface of the thigh. Remaining hip actuators (four actuators), are mounted on the body brace behind the operator's back.

Each antagonistic muscle pair attaches around an instrumented pulley, which maintains a constant defined moment arm at differing joint rotation angles. The attachment over the pulley is cable driven, which in combination with flexibility of the muscles permits tolerance for mechanical misalignments. This ability to cope with misalignments is a key feature of pMAs, which permitted lower cost in the design and construction of our exoskeletal frame. The position of each joint is sensed using a high linearity potentiometer, with torque feedback on muscle tension via strain gauges in- tegrated into the spokes of the pulleys. Depending on the activated joint, muscles of differing size are used to produce propellant motion. In general, muscles are of $30 \mathrm{~mm}$ diameter, with an 'at rest' length ranging from $350 \sim 600 \mathrm{~mm}$.

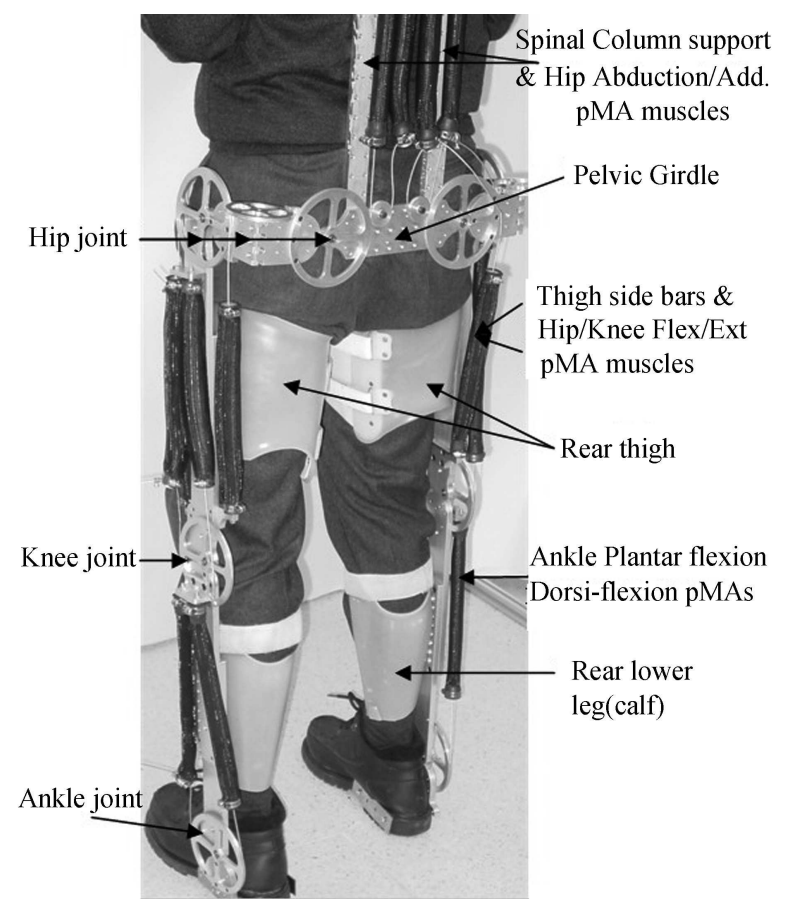

Fig. 2 Author wearing the lower body exoskeleton

Performance specification for the joints of a human leg are shown in Table $1^{[21]}$, together with maximum joint torque and range of motion. Data of the joint torques and range limits for the exoskeleton are also recorded in Table 1 for comparison. For the exoskeleton the ranges can be mechanically adjusted to suit a user's comfort limits.

Table 1 The performance and range of motion of a "normal" human, versus a pMA powered exoskeleton device

\begin{tabular}{lll}
\hline $\begin{array}{l}\text { JOINT / SEGMENT } \\
\text { Movement }\end{array}$ & $\begin{array}{l}\text { Human Isometric } \\
\text { Strength/Range }\end{array}$ & $\begin{array}{l}\text { Exoskeleton Joint } \\
\text { Torque/Range }\end{array}$ \\
\hline Hip & & \\
Flexion/Extension & $110 \mathrm{~N} \cdot \mathrm{m} 120^{\circ} / 20^{\circ}$ & $60 \mathrm{~N} \cdot \mathrm{m} 135^{\circ} / 45^{\circ}$ \\
Adduction/abduction & $125 \mathrm{~N} \cdot \mathrm{m} 45^{\circ} / 30^{\circ}$ & $65 \mathrm{~N} \cdot \mathrm{m} 135^{\circ} / 135^{\circ}$ \\
Internal Rotation & $35^{\circ}-45^{\circ}$ & $\mathrm{Un}-$ powered $110^{\circ}$ \\
External Rotation & $45^{\circ}-50^{\circ}$ & $\mathrm{Un}-$ powered $110^{\circ}$ \\
Knee & & \\
$\begin{array}{l}\text { Flexion/Extension } \\
\text { Ankle }\end{array}$ & $72.5 \mathrm{~N} \cdot \mathrm{m} 140^{\circ}$ & $60 \mathrm{~N} \cdot \mathrm{m} 140^{\circ}$ \\
Plantar Flexion/Dorsiflex & $19.8 \mathrm{~N} \cdot \mathrm{m} 50^{\circ} / 30^{\circ}$ & $60 \mathrm{~N} \cdot \mathrm{m} 105^{\circ} / 45^{\circ}$ \\
\hline
\end{tabular}

\section{System controller/user interfaces}

Eight port $2 / 2$ pneumatic valves in an inte- 
grated package $45 \mathrm{~mm} \times 55 \mathrm{~mm} \times 55 \mathrm{~mm}$ in dimensions, and weighing less than $300 \mathrm{~g}$ (MATRIX), were used within this design, and mounted at the base of the spine. These valves can be driven and controlled at up to $200 \mathrm{~Hz}$ using a PWM signal. This provides rapid and smooth motion. By incorporating a pressure sensor into valve inlets, closed loop pressure control is also possible.

Pulsing of valves, along with data collection from position, pressure, and torque sensors, is controlled from a local dedicated microcontrollers (ATMEGA8), with $\mathrm{I} / \mathrm{O}, \mathrm{ADC}$, and communication port facilities as shown in Fig. 3. An external PC is used to supervise working conditions for the prototype.

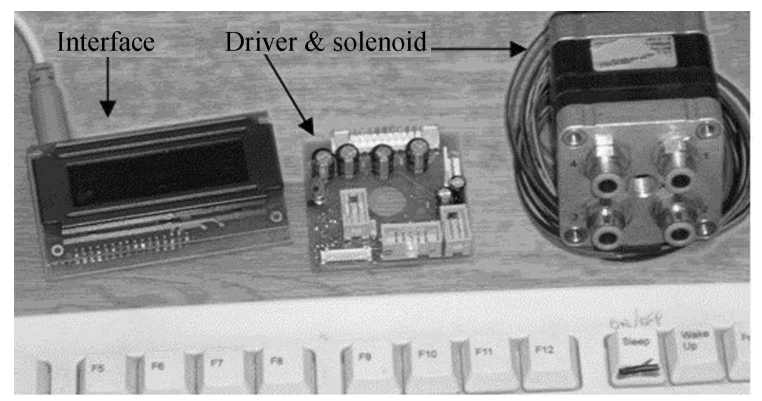

Fig. 3 A hub with an interface keyboard, and a microcontroller board with valve drivers

Each individual muscle pair (joint) is controlled by a local micro-controller (Motorola MC68HC811), which mates with a valve assembly for compact operation. Each MCU runs at $2 \mathrm{MHz}$, and can control up to 8 pMAs ( 8 inlets +8 outlets) with the joint torque control scheme illustrated in Fig. 4.

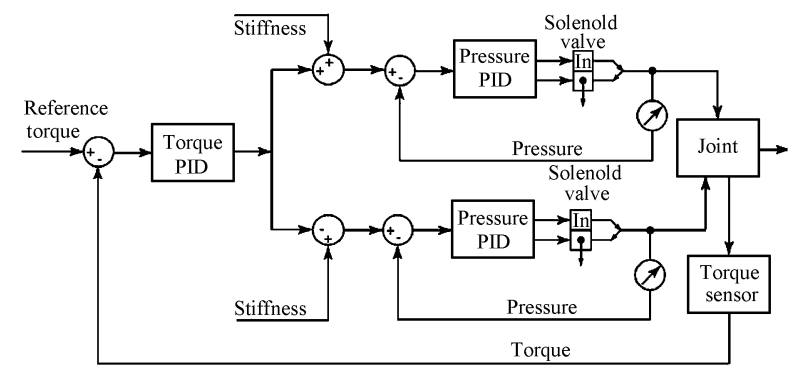

Fig. 4 A 3 level PID joint torque control scheme

Each antagonistic pair is controlled by a simple PID feedback controller on all joints. As muscles operate in pairs, the value provided by the controllers is added to one of the muscles and subtracted from its antagonist counterpart.

MCUs are connected via a serial data bus to a central controller or HUB. The hub consists of one microcontroller, an Atmel ATMEGA128, with 2 functions. First, the hub coordinates all valve control units and feeds them with self-generated data based on the exoskeleton's operating mode. Second, the hub can be used as an interface between a PC and the valve controllers. In this case, all inputs are generated by the PC. Communication between the hub and PC is completely wireless (using BlueTooth technology). PC based interface and data operation software was developed using Matlab Simulink and real time functions.

\section{$5 \quad$ Experimental results}

A series of joint operational tests were conducted to determine the "stability" of the control system and actuators, as well as orthosis structure dynamics.

Fig. 5(a) illustrates a fast step response, with a rise time of 0.3 seconds, and an overshoot of $6 \%$. Fig. 5(b) displays gauge pressure inside two pneumatic actuators during a low frequency step response.

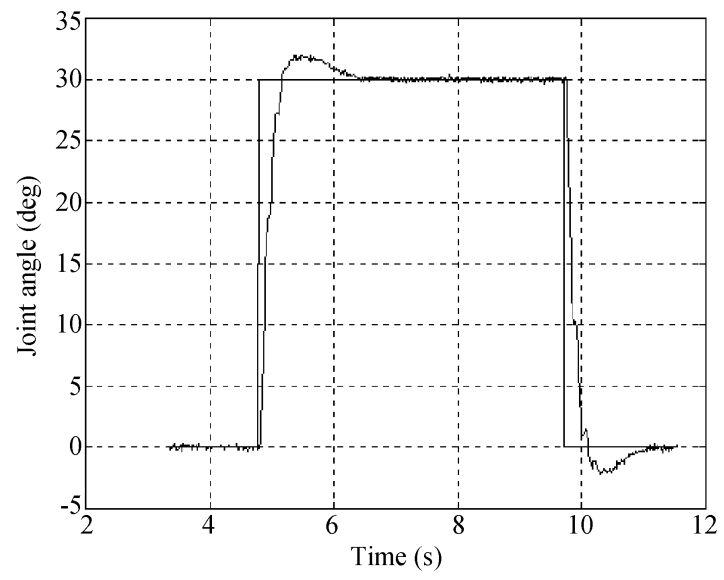

(a) System step response

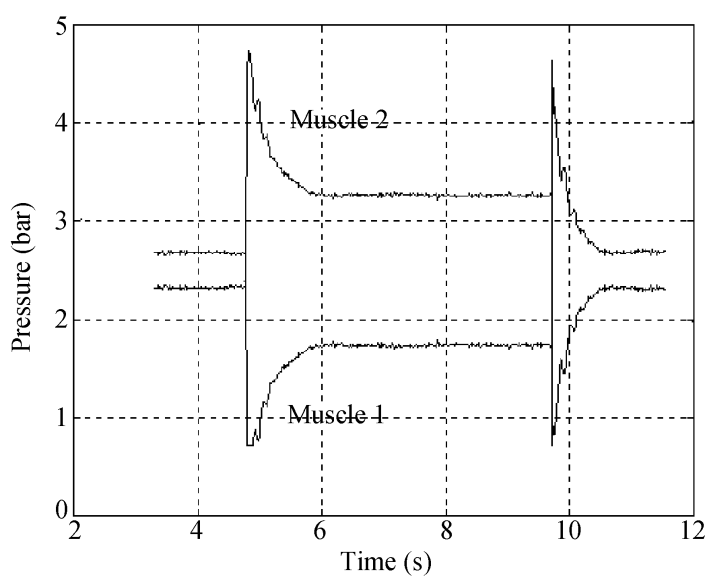

(b) Muscle effort

Fig. 5 System step response and muscle effort

In a second experiment, the sensitivity and response of the system to load variation was explored. Fig. 6(a) shows the position of a settled joint, initially around a desired position of $\theta_{d}=30 \mathrm{deg}$. At time $\approx 39 \mathrm{sec}$ a nega- 
tive disturbance load was applied to the joint $(\sim 5 \mathrm{~N} \cdot \mathrm{m})$. As can be seen in Fig. 6(a), the joint initially moved away from the desired position due to the disturbance load. The control scheme responded by increasing the pressure in muscle 2 , and reducing pressure in muscle 1. As can be seen in Fig. 6(b), the joint rapidly settled back to the desired position. At time $\approx 40.8$ seconds the disturbance load was removed. The joint position again initially moved away from, but quickly moved back to and settled again at the desired position. These experimental results reveal that the control scheme has a good ability to cope with load variation.

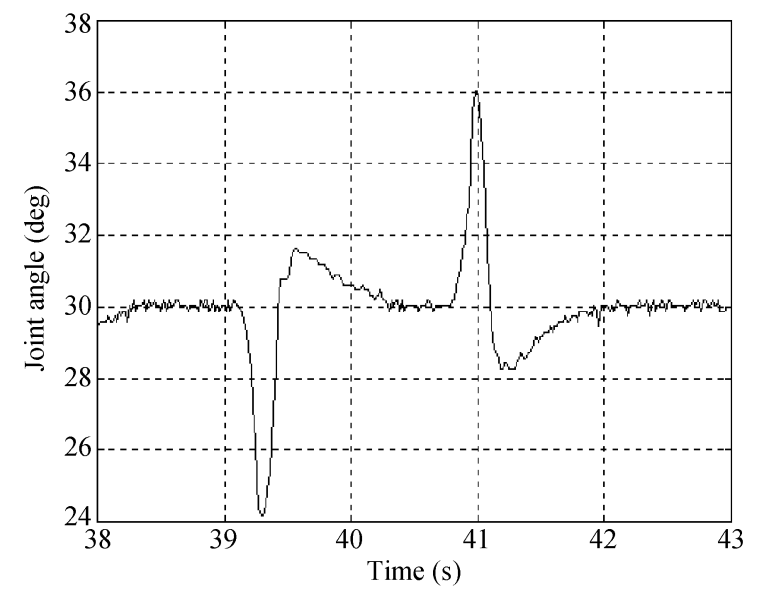

(a) System response to load disturbance

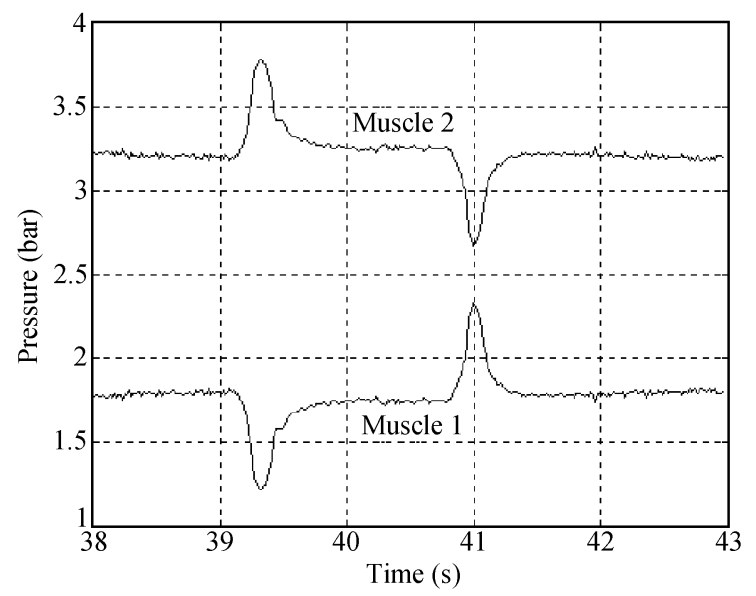

(b) Muscle effort

Fig. 6 System response to load disturbance and muscle effort

A chirp signal with different amplitudes and frequencies, as shown in Fig. 7(a) and (b), was used to evaluate the closed loop frequency response of each joint. This type of testing signal has a large range of frequency components, which helps to automate and significantly speed-up the system testing procedure. The purpose of these tests was to draw conclusions about the feasibility of using the exoskeleton for physiotherapy, rehabilitation, and training.

For each joint, two sets of input drive amplitudes were used $\left( \pm 12^{\circ}\right.$ and $\left.\pm 25^{\circ}\right)$. Tests were conducted unloaded, i.e. only the weight of the orthosis; and loaded, i.e. with limbs loaded with masses equivalent to those of human limbs, as shown in Fig. 1.

For the ankle, which has the lightest load, the frequency test range swept from 0 to $4 \mathrm{~Hz}$ in $100 \mathrm{~ms}$ steps over a specified period of $t=180 \mathrm{~s}$. For the knee, the peak frequency of the chirp was reduced to $1.5 \mathrm{~Hz}$ over a period $t=90 \mathrm{~s}$. The hip also had a $1.5 \mathrm{~Hz}$ peak frequency over $t=90 \mathrm{~s}$ when unloaded, but was reduced to a peak of $1 \mathrm{~Hz}$ when tested with the normal weight of a human limb.

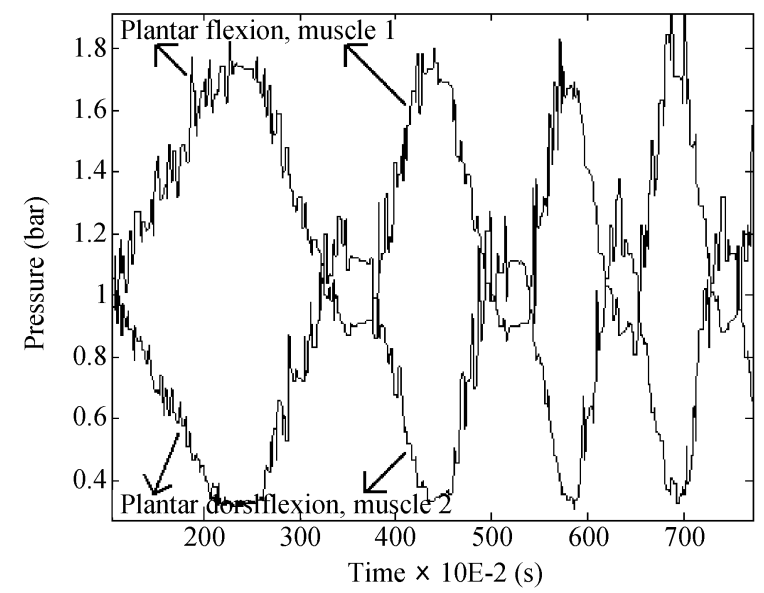

(a) Pressure response of antagonistic muscles on the right ankle

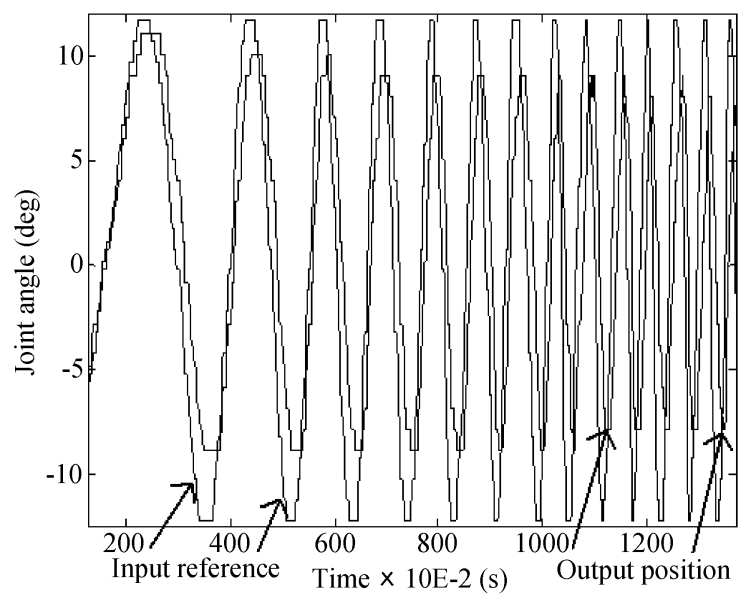

(b) Output response to a reference signal 


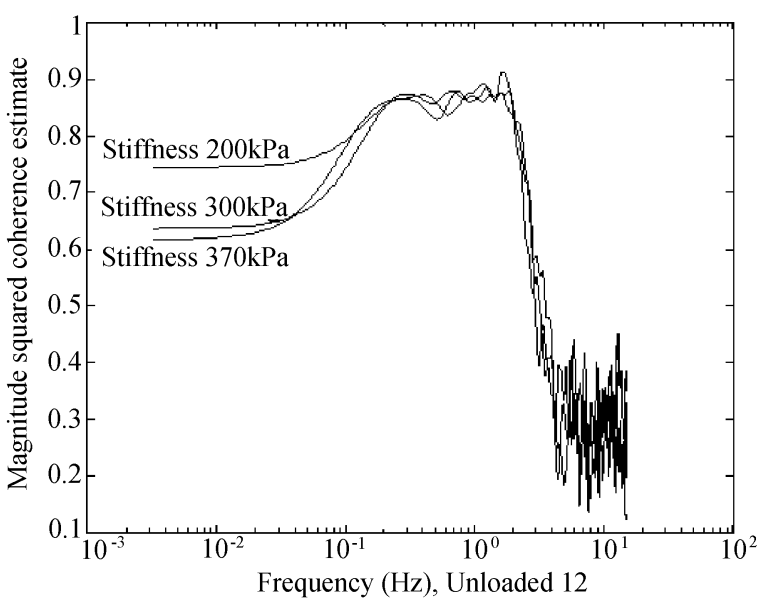

(c) Coherence ratio measures

Fig. 7 Pneumatic muscles actuator performance

Fig. 7(a) shows a typical antagonistic muscle effort during a test sequence (as illustrated by plantar and dorsi flexion of the right ankle over a $\pm 12^{\circ}$ motion with a chirp drive signal). Fig. 7(b) illustrates how well the implemented closed loop control scheme was able to track the reference motion (on the same joint), and compensate for actuator response limitations (e.g. actuator saturation, transport delay, and pulley shape/centre location). Coherence ratios for the right ankle are presented in Fig. 7(c), and show that stiffness limits the ability of the control system to transfer energy or power from the input reference to its output when responding to an excitation signal. Higher stiffness as well as loading of the limb joint with human like weight, usually generates worse coherence, as the system encounters more difficulty in responding, and loses more power. This is particularly noticeable for frequencies below $0.1 \mathrm{~Hz}$, where existing friction (static and dynamic), plays an important role. Interestingly, for frequencies between $0.1 \mathrm{~Hz}$ and $2.5 \mathrm{~Hz}$, stiffness doesn't seem to have such a pronounced effect, resulting in less differentiation. In essence, friction becomes less important at more natural dynamic excitation frequencies. Above $2.5 \mathrm{~Hz}$, and independently of stiffness, coherence always shows a sharp drop as power loss is high (and has a low coherence value as the system blocks these frequencies due to the pMAs construction characteristics). Occasional non-linearities are present, but these seem to have little disturbance effect on the results. We believe these non-linearities are mainly due to the mechanical construction of the orthosis, including the attachment of pre-tensioned muscles and cable effects. For all joints, coherence results are consistent; these results will not be analysed in depth in this paper.

Using Matlab Fast Fourier Techniques, a Transfer Function Estimate (TFE) was computed by averaging several cross powered spectral densities ${ }^{[22]}$. A suit- able Kaiser window with overlapping sample points was used to reduce the impact of leakage that different sets of window data filtering techniques generate; especially on transition edges ${ }^{[23]}$. This window was chosen from the Matlab signal processing toolbox, as it offers more freedom than other techniques ${ }^{[24]}$ (i.e. to adjust the number of points and the Beta value that forms the overlap) ${ }^{[25]}$. The choice of Kaiser window represents a compromise between the need to increase resolution and fidelity by improving the number of points used in the TFEstimate, and computational load. An increased number of averages is needed such that the number of points sampled per window is sufficient to limit noise interference.

In parallel, a coherence function was used to determine the quality of the TFE. This coherence was a function of frequency with values between 0 and 1 , which indicated how well the input corresponded to output at each frequency, as shown in Fig. 7(c). Tests were conducted and plotted at three different joint stiffnesses, corresponding to steady state values of muscle pressure in each antagonistic muscle of $200 \mathrm{kPa}(395 \mathrm{~N} / \mathrm{m})$, $300 \mathrm{kPa}(590 \mathrm{~N} / \mathrm{m})$, and $370 \mathrm{kPa}(730 \mathrm{~N} / \mathrm{m})$. It can be seen that within excitation frequencies the TFE is accurate (with coherence values normally well above 0.6). The TFE and Coherence function were implemented in Matlab (30Hz sampling frequency) for all joints of the orthosis, under different test conditions: amplitudes, time, frequency range, and stiffness. It was important to ensure that both functions were operational under the same data conditions.

For all plotted data graphs, the Nyquist frequency was half the sampling frequency, as shown on all XAxes. To avoid effecting and disturbing the results, all DC sensor levels were removed using a "dtrend" Matlab function so that their average became 0 . The smallest frequency we could measure depended on how good excitation signals were, and how the tests were carried out.

Figs. 8 and 9 show the frequency responses of the right ankle and knee joints for motions of $\pm 12^{\circ}$ and $\pm 25^{\circ}$. Responses were recorded under 3 different muscle stiffness conditions as outlined above. In figures (a) and (b) of each sequence, the joints were unloaded. In figures (c) and (d) the joints were loaded with weights to simulate normal limb weight. Figures (e) and (f) show the phase for each of the joints.

Fig. 10 shows the frequency responses of the hip joint for chirp signal motions of $\pm 12^{\circ}$ and $\pm 25^{\circ}$. Due to limb loading, higher drive forces are required, which means that tests were not conducted for the lowest stiffness model. Test stiffness was $(300 \mathrm{kPa}$ and $370 \mathrm{kPa}$ ) when unloaded, and only the highest stiffness of $(370 \mathrm{kPa})$ when loaded. Fig. 10 (a) shows motion 


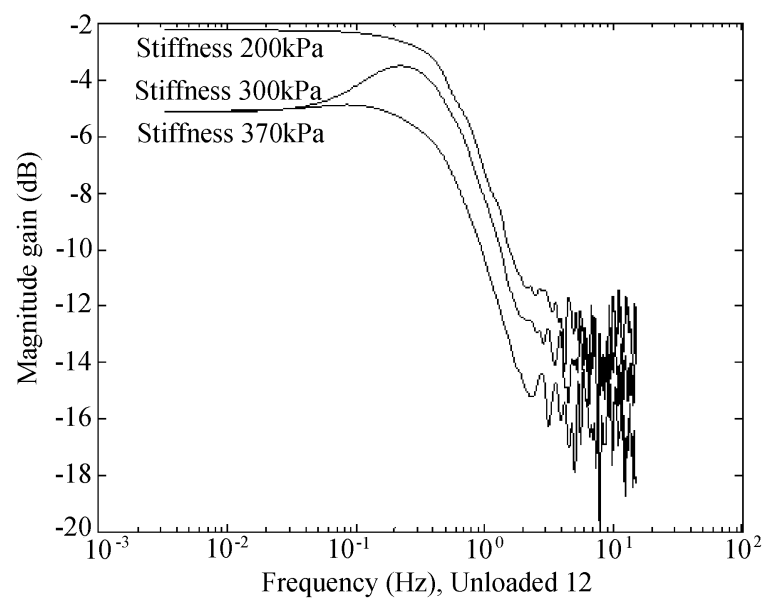

(a) Right ankle $\pm 12^{\circ}$ unloaded

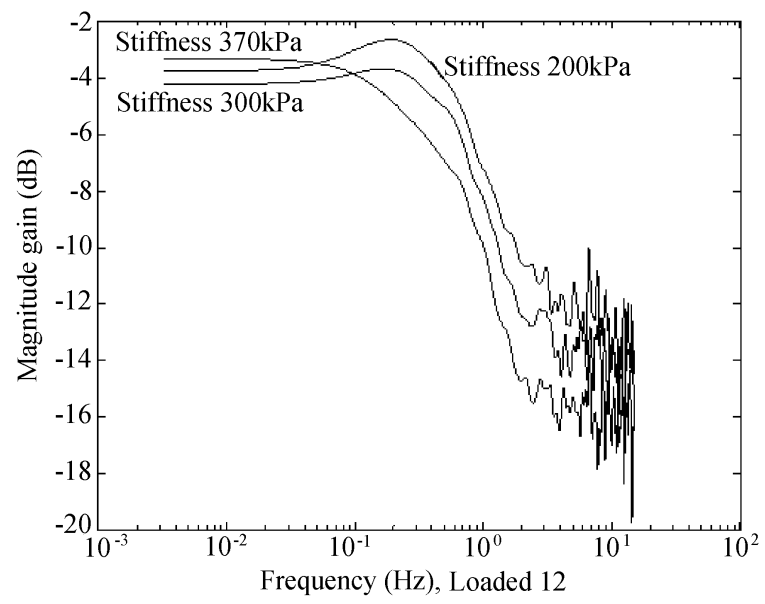

(c) Right ankle $\pm 12^{\circ}$ loaded

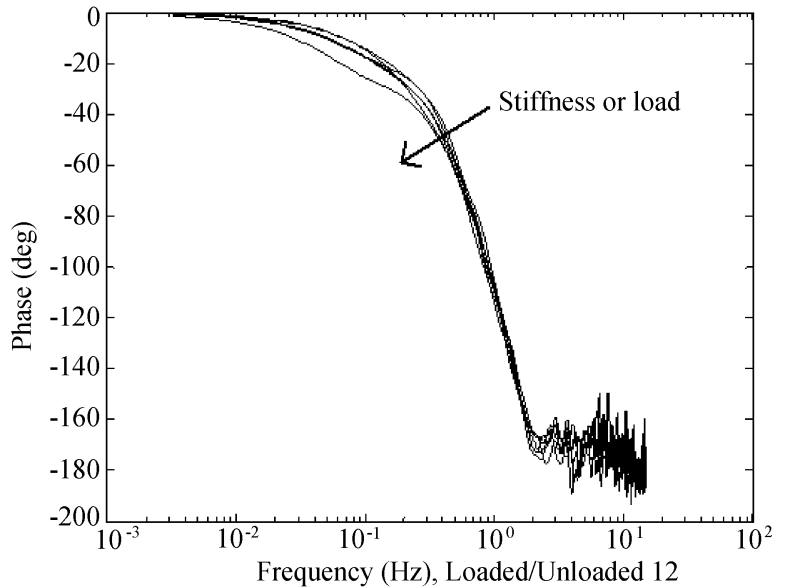

(e) Right ankle phased $\pm 12^{\circ}$ loaded \& unloaded

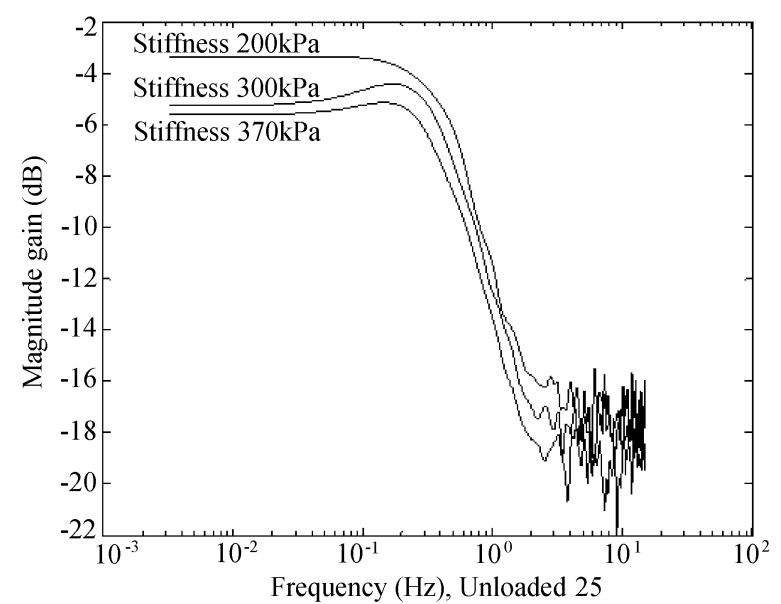

(b) Right ankle $\pm 25^{\circ}$ unloaded

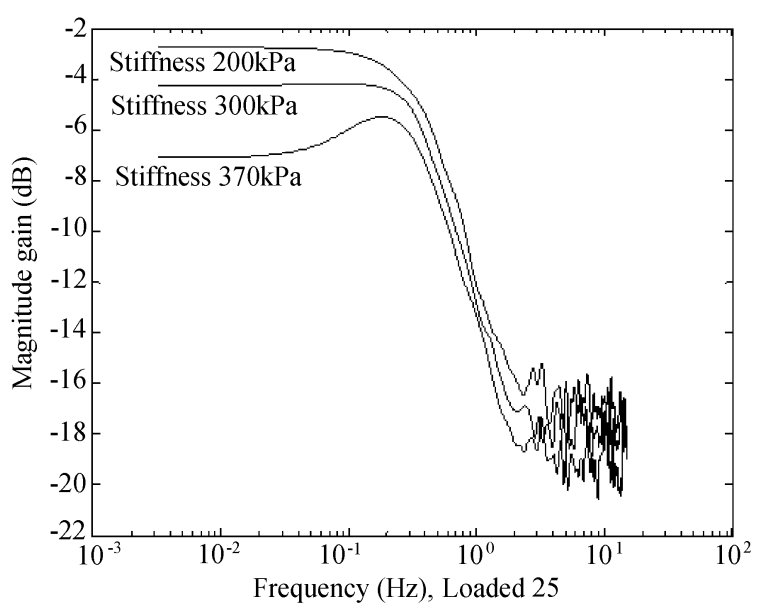

(d) Right ankle $\pm 25^{\circ}$ loaded

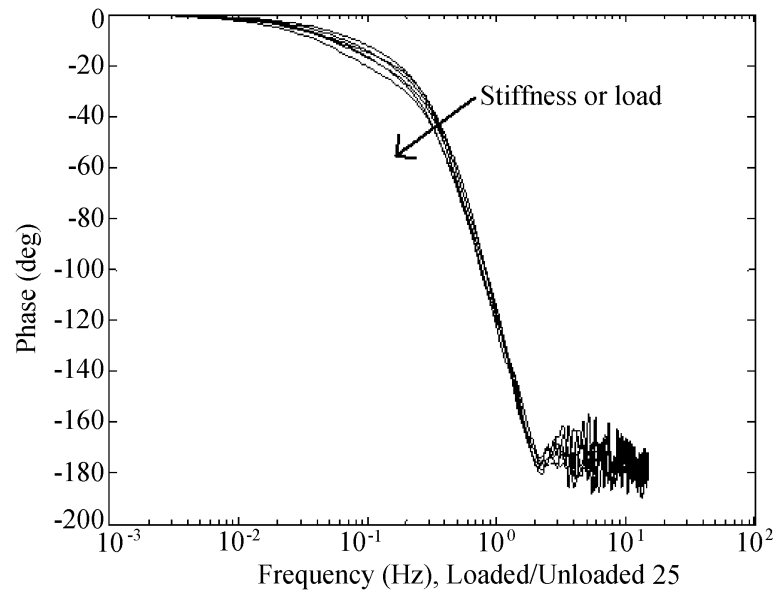

(f) Right ankle phased $\pm 25^{\circ}$ loaded \& unloaded

Fig. 8 Right ankle TFE unloaded, loaded, and phases 


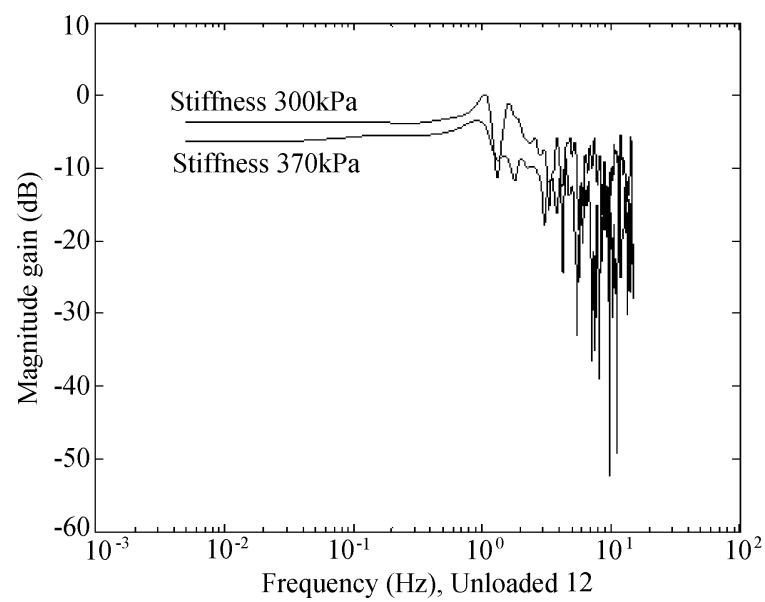

(a) Right knee $\pm 12^{\circ}$ unloaded

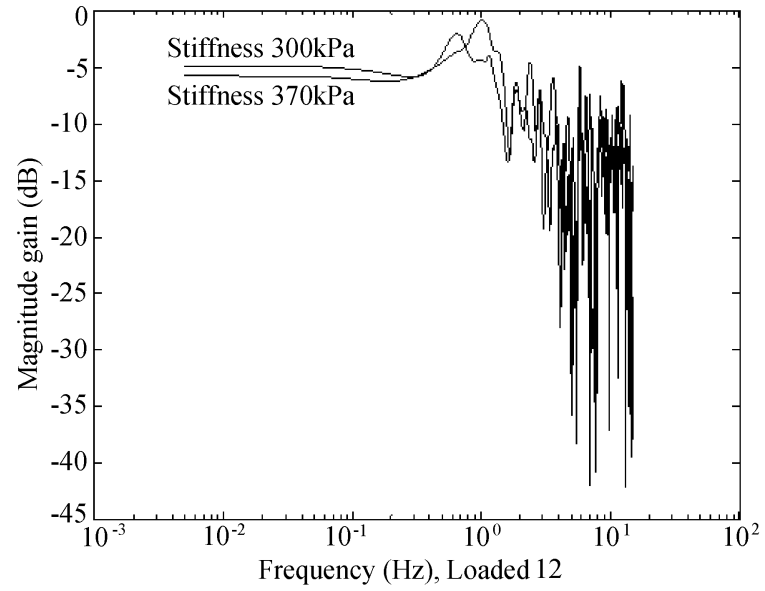

(c) Right knee $\pm 12^{\circ}$ loaded

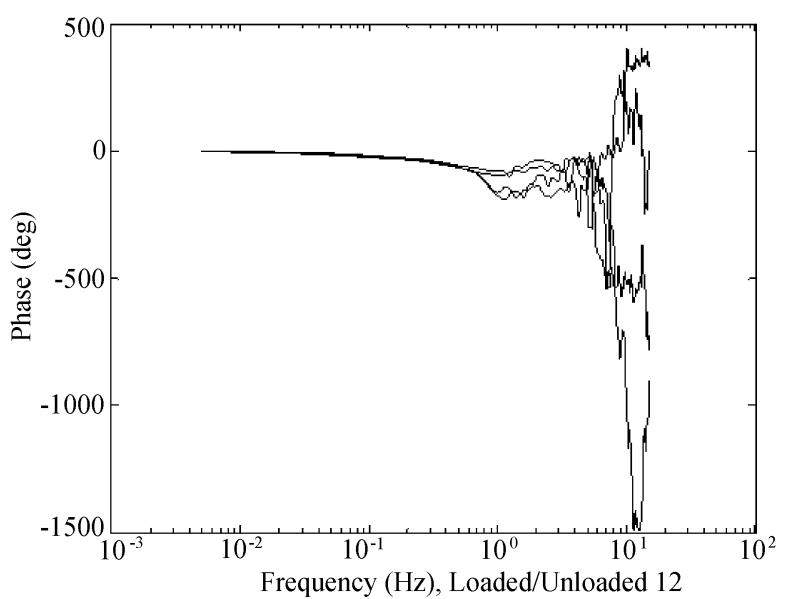

(e) Right knee phases $\pm 12^{\circ}$ loaded \& unloaded

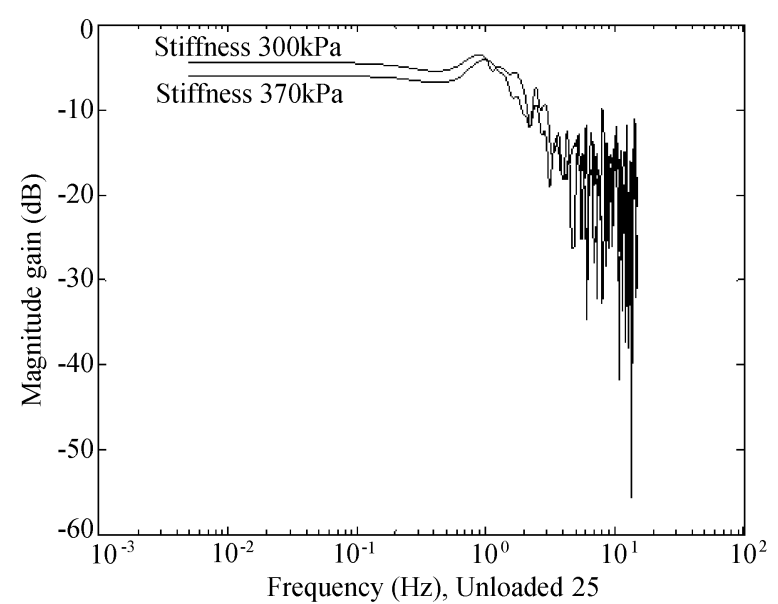

(b) Right knee $\pm 25^{\circ}$ unloaded

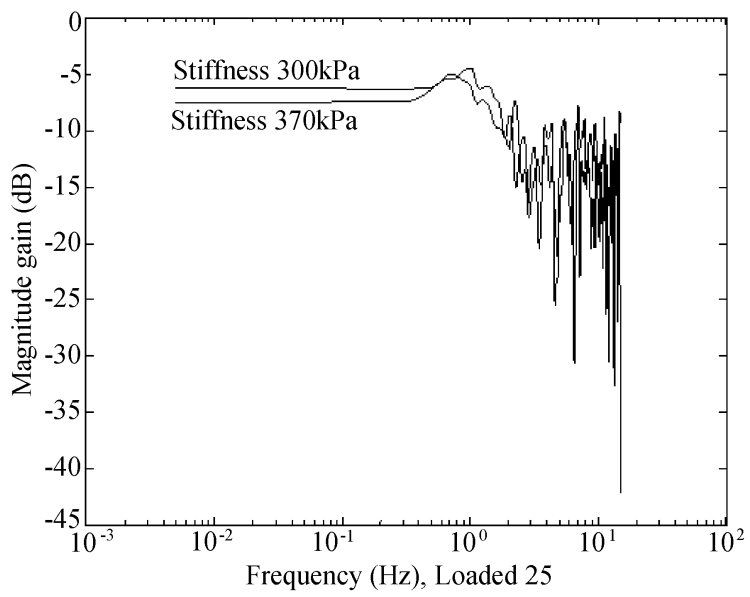

(d) Right knee $\pm 25^{\circ}$ loaded

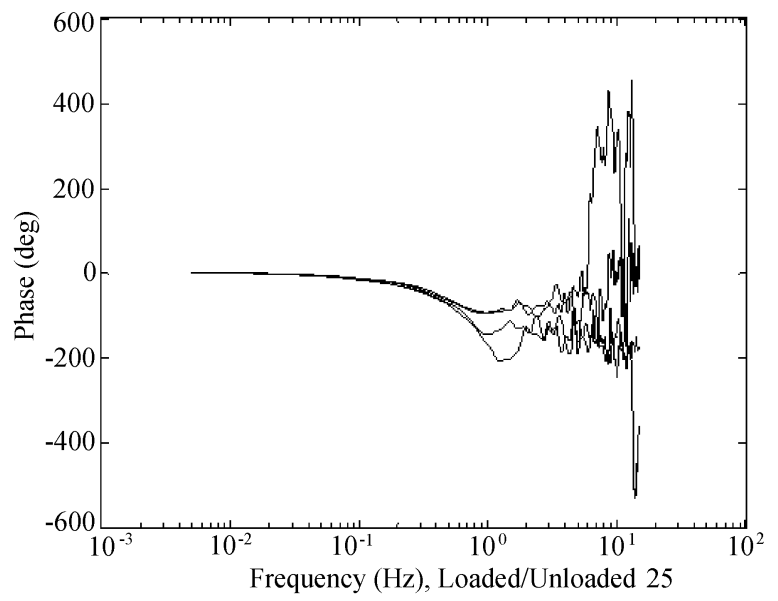

(f) Right knee phases $\pm 25^{\circ}$ loaded \& unloaded

Fig. 9 Right knee TFE unloaded, loaded, and phases 


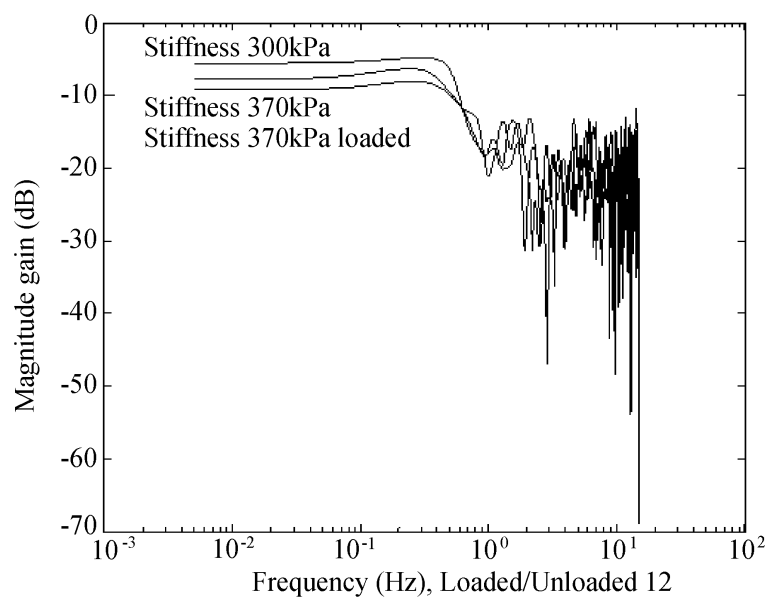

(a) Right hip phased $\pm 12^{\circ}$ loaded \& unloaded

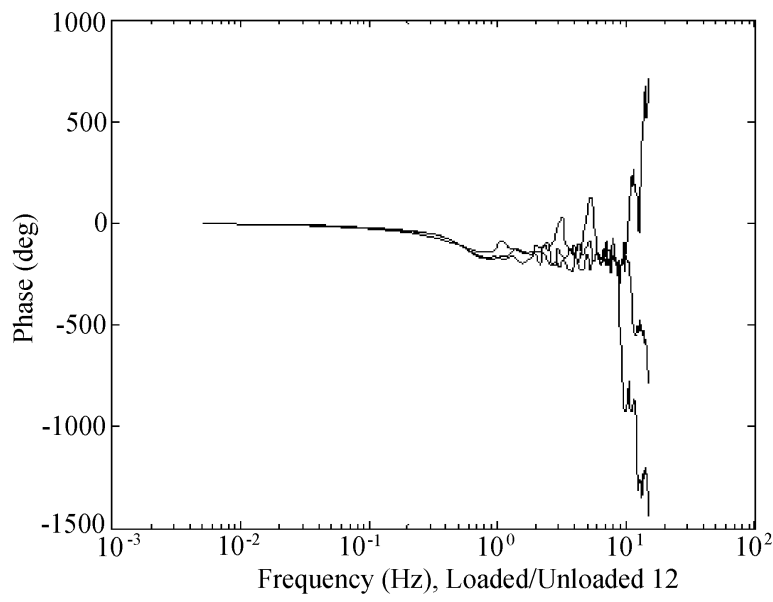

(c) Right hip phased $\pm 12^{\circ}$ loaded \& unloaded

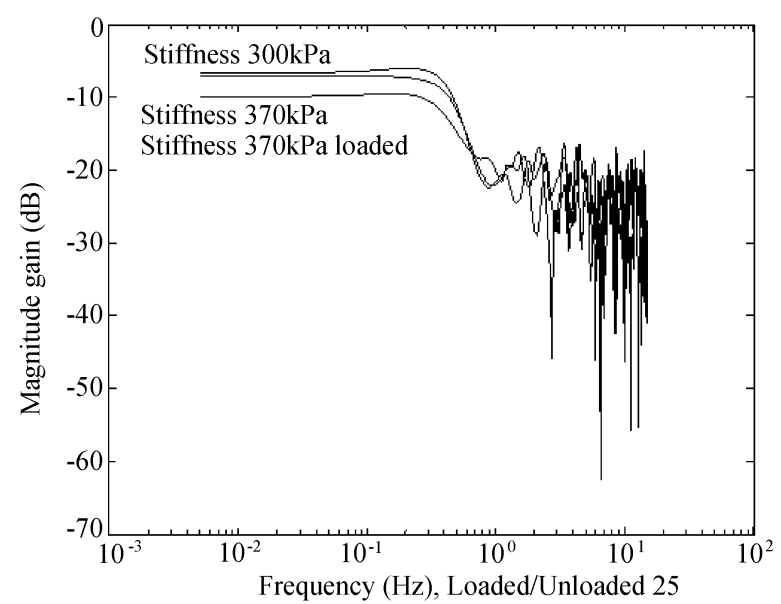

(b) Right hip phased $\pm 25^{\circ}$ loaded \& unloaded

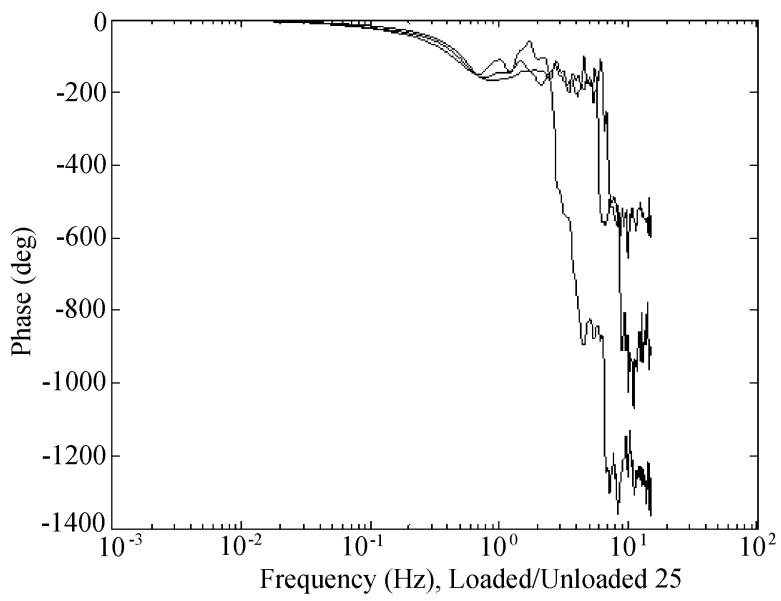

(d) Right hip phased $\pm 25^{\circ}$ loaded \& unloaded

Fig. 10 Hip TFE unloaded at left, and loaded at centre

over $\pm 12^{\circ}$, and (b) motion for $\pm 25^{\circ}$. The three plots recorded in each graph show the unloaded $(300 \mathrm{kPa}$ and $370 \mathrm{kPa}$ ), and the loaded response at a stiffness of $370 \mathrm{kPa}$. Figures (c) and (d) show the phase response for the same scenario.

Careful analysis of the experimental data results, shows that:

- Phase plots show a lag which follows a characteristic profile (typical of a $1^{\text {st }}$ order system).

- Stiffness contributes positively to increase the phase at lower frequencies, as the system becomes slower. It also causes the system to lose energy with lower Magnitude Gain.

- On average and surprisingly, the coherence values for low frequencies at the Knee and Hip joints (both unloaded and loaded), are better than for the Ankle. It is believed that this is a pendulum like natural frequency resonance behaviour, which seems to improve the results.

- This natural resonance frequency is estimated to be approximately $0.7 \mathrm{~Hz}$ (similar to a human gait). This is shown by the inflection point near this transition point.

- For similar stiffness, the loaded Knee and Hip joints may present a similar or even better Phase, as the system helped by the resonant pendulum effect seems to track the input reference more rapidly.

- Especially for the Knee and more noticeably for the Hip, the Magnitude Gain shows that the control system follows $\pm 12^{\circ}$ motions more easily than the $\pm 25^{\circ}$ angle motion span. This is due to the non linear actuator force power profile.

- Occasional non-linearities are present, but seem to have lower disturbance effect in Magnitude Gain and Phase results than stiffness. We believe these nonlinearities are mainly due to mechanical considerations, including the attachment of pre-tensioned muscles and cables. Structural vibration during the testing trials also contributed to unexpected starting conditions that are more noticeable below excitation frequencies 
of $0.01 \mathrm{~Hz}$.

\section{Conclusions and future work}

This work has shown how complex biologically inspired structures can be constructed and powered by 'soft' actuators, which macroscopically have many characteristics similar to natural muscle, while still retaining the beneficial attributes of conventional mechanical systems.

Overall response, muscle effort, ability to handle load disturbance, and human gait systemtracking/guiding capabilities, achieved with a healthy individual (to ensure stability and safety), revealed that this system may be successfully used for some medical conditions involving degenerative muscle wasting diseases/weak lower limb muscles, or conditions resulting in reduced coordination of human motor control.

The current performance in terms of joint response, while being at a lower level than achievable by an able bodied user, is more than adequate for the rehabilitation areas for which it is designed.

At the same time, on-going development and actuator research suggests that performance can be increased by several hundred percent. The full implementation of these new developments will form part of a future research program.

Future work will investigate further the use of this structure in power assisting modes. Key developments will include:

i) Enhanced power outputs from actuators to equal the power of human leg muscles.

ii) Integration of the exoskeleton into a full body support kit based around a treadmill walker.

iii) Continued testing and validation with healthy test subjects.

iv) Testing with subjects suffering from muscle wastage or paralysis.

The use of the design philosophy outlined in this paper, and in particular the utilisation of a soft actuation system, provides a system with an inherent safety and dependability profile that cannot easily be achieved with conventional designs, and may provide a valuable insight into the development of powered assistive devices.

\section{References}

[1] Audit Commission, Audit Commission Update, Fully Equipped 2002 assisting independence. Audit Commission for Local Authorities and the National Heath Service in England and Wales. London ISBN 1862403678, 2002.

[2] R. Waters, B. Lunsford. Energy Cost of Paraplegic Locomotion. Journal of Bone Joint Surgery Am, vol. 67, no. 8, pp. 1245-1250, 1995.
[3] K. R. Kaufman, S. E. Irby, J. W. Mathewson, R. W. Wirta, D. H. Sutherland. Energy-Efficient Knee-Ankle-Foot Orthosis: a Case Study. Journal of Prosthetics and Orthotics, vol. 8, no. 3, pp. 79-85, 1996.

[4] M. Muccio, B. Andrews, E. Marsolais. Electronic Orthoses: Technology, Prototypes and Practices. American Academy of Orthotists and Prosthetists, vol. 1 no. 1, pp. 3-17, 1989.

[5] J. Clinkingbeard, J. Gersten, D. Hoehn. Energy Cost of Ambulation in Traumatic Paraplegia. American Journal Physics and Medicine, vol. 43, pp. 157-165, 1964.

[6] E. Marsolais, R. Kobetic. Functional Electrical Stimulation for Walking in Paraplegia. Journal of Bone Joint Surgery, vol. 69-A, pp. 728-733, 1995.

[7] H. F. M Van der Loos. Rehabilitation Mechatronic Therapy Devices. In Proceedings of Workshop on Biomedical Robotics and Biomechatronics, International Conference on Robotics and Automation 2004, New Orleans, April 2004.

[8] G. Belforte, L. Gastaldi, M. Sorli. Pneumatic Active Gait Orthosis. Mechatronics, vol. 11, pp. 310-323, 2001.

[9] P. J. Greene, M. Granat. A Knee and Ankle Flexing Hybrid Orthosis for Paraplegic Ambulation. Medical Engineering \& Physics, vol. 25, no. 7, pp. 539-545, 2003.

[10] R. Harrison, E. Lamaire, Y. Jeffreys, L. Goudreau. Design and Pilot Testing of an Orthotic Stance-Phase Control Knee Joint. Orthopadie-Technik, vol. 111, pp. 2-4, 2001.

[11] H. F. M. Van der Loos. Rehabilitation Mechatronic Therapy Devices. In Proceedings of Workshop on Biomedical Robotics and Biomechatronics, International Conference on Robotics and Automation 2004, New Orleans, 2004.

[12] T. Suga, O. Kameyama, R. Ogawa, M. Matsuura, H. Oka. Newly Designed Computer Controlled Knee-Ankle-Foot Orthosis (Intelligent Orthosis). Prosthetics and Orthotics International, vol. 22, no. 3, pp. 230-239, 1998.

[13] E. Saitoh, T. Suzuki, S. Sonoda, J. Fujitani, Y. Tomita, N. Chino. Clinical Experience with a New Hip-Knee-AnkleFoot Orthotic System Using a Medial Single Hip Joint for Paraplegic Standing and Walking. American Journal Physics Medical Rehabilitation, vol. 75, no. 3, pp. 98-203, 1996.

[14] E. Genda, K. Oota, Y. Suzuki. A New Walking Orthosis for Paraplegics: Hip and Ankle Linkage System. Prosthetics \& Orthotics International, vol. 28, no. 1, pp. 69-74, 2004.

[15] D. Ferris. An Improved Ankle Foot Orthosis Powered by Pneumatic Muscles. In Proceedings of International Society of Biomechanics XIXTH Congress The Human Body in Locomotion, Auckland, New Zealand, 2003.

[16] S. Hawran, F. Biering-Sorensen. The Use of Long Leg Callipers for Paraplegic Patients: a Follow-up Study of Patients Discharged 1973-1982. Spinal Cord, vol. 34, no. 11, pp. 666-668, 1996.

[17] O. Khatib. A New Actuation Approach for Human Friendly Robot Design. In Proceedings of 3rd International Advanced Robotics Programme (IARP) Workshop on Dependable Robots in Human Environments, Manchester, England, Sept. 2004.

[18] Davis, N. Tsagarakis, J. Canderle, D. G. Caldwell. Enhanced Modelling and Performance in Braided Pneumatic Muscle Actuators. International Journal of Robotics Research, vol. 22, no. 3-4, pp. 213-227, 2003.

[19] N. Tsagarakis. Integrated Haptic Interface: Tactile and Force Feedback for Improved Realism in VR and Telepresence Application. Ph.D dissertation, University of Salford, 2000.

[20] D. Caldwell, D. Medrano-Cerda, M. Goodwin. Control of Pneumatic Muscle Actuators. IEEE Control Systems Magazine, vol. 15, no. 1, pp. 40-48, 1995.

[21] Human Engineering Guide to Equipment Design. American Institutes for Research, Washington D.C. 1972

[22] Matlab 7.0 (R14), The Mathworks Inc, 2005. 
[23] A. V. Oppenheim, R. W. Schafer. Discrete-Time Signal Processing. Prentice-Hall, NJ, USA, pp. 453, 1989.

[24] Selected Papers in Digital Signal Processing II. IEEE Press, New York, 1975.

[25] J. F. Kaiser. Nonrecursive Digital Filter Design Using the Sinh Window Function. In Proceedings of 1974 IEEE Symposium Circuits and Systems, pp. 20-23, 1974.

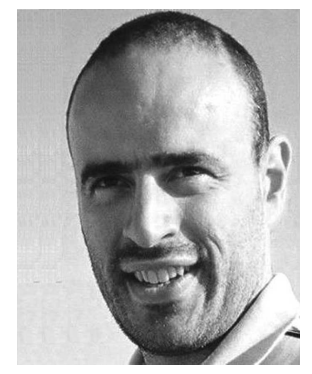

Nelson Costa received his B.Sc degree in Computer and Electronic Engineering in 1995 and his M.Sc in Robotic Systems and Automation in 1999, both at the University of Coimbra, Portugal. He is currently pursuing his Ph.D. in Advanced Robotics at the University of Salford, Greater Manchester, UK.

His research interests include sensors, actuators, power sources, force augmentation exoskeletons, rehabilitation robotics and haptic feedback systems.

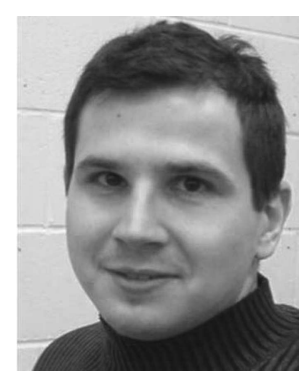

Milan Bezdicek received his M.Sc in Mechatronics from Brno University of Technology in Czech Republic in 2003. He is currently Ph.D. student of Robotics and haptics at the University of Salford, UK.

His research interests include biomechanics, electronics, computer controlled machining, artificial neural networks, haptics, DSP, smart sensors and structure analyzing.

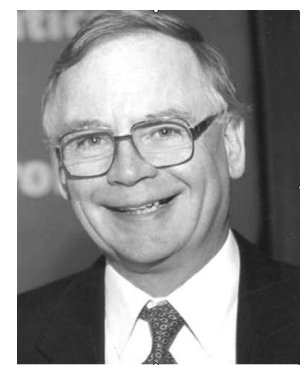

John O. Gray obtained his Ph.D. and D.Sc degrees from the University Manchester in control engineering and his early work focussed on aspects of non linear control systems, C.A.D procedures and precision electromagnetic instrumentation. In 1988 with academic and industrial colleagues he established the U.Ks National Advanced Robotics Research Centre. In the 1990s he organised a series of EC.spon-

sored conferences on advanced robotics and more recently (20012005) chaired EPSRC and IEE networks on advanced robotics.

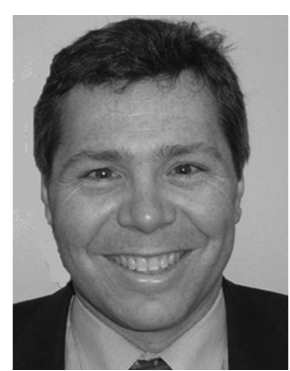

Darwin G. Caldwell received his B.Sc and Ph.D. in Robotics from the University of Hull in 1986 and 1990 respectively. In 1994 in received a M.Sc in Management. Since 1999 he has been Chair of Advanced Robotics in the Centre for Robotics and Automation at the University of Salford.

$\mathrm{He}$ is the author or co-author of over 150 academic papers and has 4 patents. His research interests include innovative actuators and sensors, haptic feedback, force augmentation exoskeletons, dexterous manipulators, humanoid robotics, biomimetic systems, rehabilitation robotics and robotic systems for the food industry.

Prof. Caldwell is currently chair of the UKRI region of the IEEE (Robotics and Automation Society).

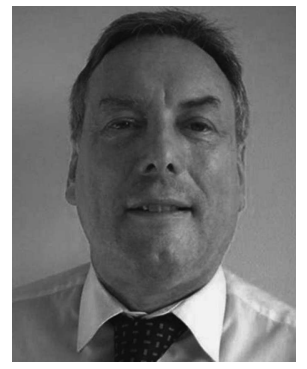

claudication.

He has a number of UK patents on orthotic knee joint design. His research interests include all aspects of lower limb orthotics and footwear design.

Stephen Hutchins is Senior Lecturer in Orthotics at the University of Salford and a registered orthotist with HPC having graduated as an orthotist in 1978. He obtained an M.Sc in Orthotics by research in 1997 for the design of prototype orthotic knee joints to be used in prophylactic knee bracing. He is currently pursuing his Ph.D. on the subject of rocker sole development in the treatment of intermittent 